\title{
LncRNA HSPA7 in human atherosclerotic plaques sponges miR-223 and promotes the proinflammatory vascular smooth muscle cell transition
}

\author{
Soo-jin Ann ${ }^{1}$, Hyoeun Bang ${ }^{2}$, Chan Joo Lee ${ }^{3}$, Jaewon Oh $^{3}$, Sungha Park ${ }^{3}$, Seok-Min Kang (iD ${ }^{3}$, Jung Kyoon Choi (iD ${ }^{2}$ and \\ Sang-Hak Lee iD ${ }^{3 凶}$
}

(C) The Author(s) 2021

\begin{abstract}
Although there are many genetic loci in noncoding regions associated with vascular disease, studies on long noncoding RNAs (IncRNAs) discovered from human plaques that affect atherosclerosis have been highly limited. We aimed to identify and functionally validate a IncRNA using human atherosclerotic plaques. Human aortic samples were obtained from patients who underwent aortic surgery, and tissues were classified according to atherosclerotic plaques. RNA was extracted and analyzed for differentially expressed IncRNAs in plaques. Human aortic smooth muscle cells (HASMCs) were stimulated with oxidized low-density lipoprotein (oxLDL) to evaluate the effect of the identified IncRNA on the inflammatory transition of the cells. Among 380 RNAs differentially expressed between the plaque and control tissues, IncRNA HSPA7 was selected and confirmed to show upregulated expression upon oxLDL treatment. HSPA7 knockdown inhibited the migration of HASMCs and the secretion and expression of IL-1 $\beta$ and IL-6; however, HSPA7 knockdown recovered the oxLDL-induced reduction in the expression of contractile markers. Although miR-223 inhibition promoted the activity of Nf-KB and the secretion of inflammatory proteins such as IL-1 $\beta$ and IL-6, HSPA7 knockdown diminished these effects. The effects of miR-223 inhibition and HSPA7 knockdown were also found in THP-1 cell-derived macrophages. The impact of HSPA7 on miR-223 was mediated in an AGO2-dependent manner. HSPA7 is differentially increased in human atheroma and promotes the inflammatory transition of vascular smooth muscle cells by sponging miR-223. For the first time, this study elucidated the molecular mechanism of action of HSPA7, a IncRNA of previously unknown function, in humans.
\end{abstract}

Experimental \& Molecular Medicine (2021) 53:1842-1849; https://doi.org/10.1038/s12276-021-00706-8

\section{INTRODUCTION}

The risk of atherosclerotic cardiovascular diseases, such as coronary artery disease, is strongly attributable to genetic factors ${ }^{1}$. According to recent genetic studies, many novel genetic loci are associated with coronary artery disease but have an unknown function. In addition, many of them are reportedly located in noncoding regions of the human genome ${ }^{2}$. For example, the association between the 9p21 locus and myocardial infarction has been replicated by research groups. ANRIL, a noncoding RNA, was found to be located in this locus, and its function in influencing cell proliferation was partly elucidated ${ }^{3}$.

Long noncoding RNAs (IncRNAs) are the longest types of noncoding RNAs and are differentiated from other shorter noncoding RNAs. LncRNAs have very diverse functions and are regarded as attractive therapeutic targets due to their tissue, cell, and disease specificities ${ }^{4}$. As IncRNAs affect distal targets, they stabilize ribonucleoprotein complexes, alter phosphorylation pathways, or act as competing endogenous RNAs ${ }^{5}$. Dozens of IncRNAs have been reported to affect the development of atherosclerosis. Prior studies showed that specific IncRNAs regulate cells in arteries, including endothelial cells, vascular smooth muscle cells (VSMCs), and macrophages ${ }^{4,6,7}$. For instance, IncRNA H-19 is known to increase VSMC proliferation ${ }^{8}$, whereas IncRNA-p21 inhibits it ${ }^{9}$. Although some additional IncRNAs were identified to regulate vascular cells ${ }^{10}$, IncRNAs discovered from human vascular cells that affect atherosclerosis have been highly limited. Conversely, microRNAs inhibit target mRNAs usually via degradation or translational blocking ${ }^{11}$. Some microRNAs are involved in atherosclerosis by affecting SMC proliferation or phenotypic changes ${ }^{12}$.

In the current study, we aimed to analyze human atherosclerotic plaques and identify and functionally validate a IncRNA with a previously unknown function. We challenged VSMCs with several stimuli, including oxidized low-density lipoprotein (oxLDL), a hallmark of atherosclerosis, and evaluated the effects and mechanisms of action of the identified IncRNAs.

\section{MATERIALS AND METHODS}

\section{Study subjects and aortic tissue extraction}

The study protocol (no. 4-2013-0688) was approved by the Institutional Review Board of Severance Hospital, Seoul, Korea. All participants provided

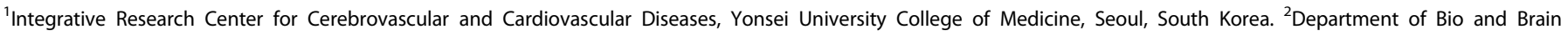

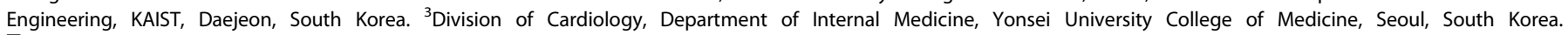
凶email: shl1106@yuhs.ac
} 
Table 1. Characteristics of study participants according to the presence of aortic atherosclerotic plaques.

\begin{tabular}{|c|c|c|c|c|c|c|}
\hline \multirow[b]{2}{*}{ Patient number } & \multicolumn{2}{|c|}{ Atherosclerosis $(n=2)$} & \multicolumn{2}{|c|}{ Control $(n=4)$} & \multirow[b]{2}{*}{5} & \multirow[b]{2}{*}{6} \\
\hline & 1 & 2 & 3 & 4 & & \\
\hline Age & 79 & 72 & 68 & 48 & 61 & 73 \\
\hline Sex & Female & Male & Male & Male & Female & Female \\
\hline Diabetes mellitus & No & No & No & No & No & No \\
\hline Hypertension & Yes & Yes & No & Yes & No & Yes \\
\hline Smoking & No & Yes & Yes & No & No & No \\
\hline Hyperlipidemia* & No & Yes & No & No & No & No \\
\hline Coronary artery disease & No & No & No & No & No & Yes \\
\hline Body mass index & 23.4 & 27.6 & 23.2 & 20.9 & 23.9 & 20.7 \\
\hline
\end{tabular}

*Defined as low-density lipoprotein-cholesterol $\geq 160 \mathrm{mg} / \mathrm{dL}$.

written informed consent. Aortic samples were obtained from patients who underwent aortic graft replacement surgery for a thoracic aortic aneurysm. Samples were classified by an experienced pathologist according to the presence of atherosclerotic plaques. The characteristics of the participants according to aortic atherosclerotic plaques are presented in Table 1. The pathologist classified the lesions according to the modified classification of the American Heart Association without any knowledge of the specimens ${ }^{13}$.

\section{RNA sequencing and analysis of IncRNAs}

RNA was extracted from cells using a Ribospin RNA Extraction Kit (GeneAll, Seoul, Korea). RNA concentration and purity were assessed using a NanoDrop ND1000 spectrophotometer. Total RNA sequencing libraries were prepared using a TruSeq RNA sample preparation kit (Illumina, San Diego, CA, USA). The fragmentation step resulted in an RNA-Seq library that included inserts of approximately $100-400 \mathrm{bp}$. The average insert size in an Illumina TruSeq library was approximately $200 \mathrm{bp}$. cDNA fragments underwent an end repair process: the addition of a single ' $A$ ' base to the 3 ' end and then ligation of adapters. Finally, the products were purified and enriched with polymerase chain reaction $(P C R)$ to create final doublestranded cDNA libraries. Libraries were quantified using KAPA Library Quantification kits for the Illumina HiSeq 2500 platform according to the protocol guide KK4855 (KAPA Biosystems, Wilmington, MA, USA). We normalized the RNA sequencing data by quantile normalization as previously described ${ }^{14}$. The differentially expressed genes between samples with and without atherosclerotic plaques were compared using Cuffdiff. Genes with upregulated and downregulated expression with $q$ values $<0.05$ and $>$ two-fold changes were identified.

\section{Cells and other reagents}

Human aortic smooth muscle cells (HASMCs) were purchased from Lonza (Basel, Switzerland) and cultured in SMC growth basal medium containing growth factor supplemented with $2 \%$ fetal bovine serum and penicillin/ streptomycin at $37^{\circ} \mathrm{C}$. THP-1 cells, a human monocyte cell line, were purchased from the Korean Cell Line Bank (Seoul, Korea). The cells were cultured in RPMI-1640 medium containing $10 \%$ fetal bovine serum and streptomycin $(100 \mathrm{U} / \mathrm{mL})$-penicillin $(100 \mu \mathrm{g} / \mathrm{mL})$ at $37^{\circ} \mathrm{C}$ with $5 \% \mathrm{CO}_{2}$. THP-1 cells were plated in six-well dishes $\left(1 \times 10^{6}\right.$ cells/well $)$ and treated with $50 \mathrm{nM}$ phorbol-12-myristate-13-acetate for $24 \mathrm{~h}$ for differentiation into macrophages. Lipopolysaccharide (LPS) and angiotensin II (Angll) were purchased from Sigma-Aldrich (St. Louis, MO, USA) for HASMC stimulation. Angiotensin II is a key peptide hormone in the renin-angiotensinaldosterone system. This molecule exerts adverse cardiovascular effects by increasing vascular tone, fluid retention, thrombosis, and inflammation $^{15,16}$. Low-density lipoprotein (LDL) for oxLDL was isolated from the plasma of healthy donors using sequential ultracentrifugation. LDL was dialyzed for $24 \mathrm{~h}$ at $4{ }^{\circ} \mathrm{C}$ with phosphate-buffered saline and oxidized for $24 \mathrm{~h}$ using $5 \mu \mathrm{M} \mathrm{CuSO}_{4}$ at $37^{\circ} \mathrm{C}$. Ethylenediaminetetraacetic acid was added to stop the reaction, and thiobarbituric acid reactive substance assays were used to analyze the oxidation state of LDL before each experiment.

\section{Migration assay}

For analysis and comparison of HASMC migration, the cells were added to the upper Transwell chamber (Neuro Probe, Inc., Gaithersburg, MD, USA) in serum-free medium after transfection with siHSPA7 or control siRNA for $24 \mathrm{~h}$. The lower chamber was filled with SMC growth basal medium with fetal bovine serum. Next, LPS $(10 \mathrm{ng} / \mathrm{mL})$, oxLDL $(50 \mu \mathrm{g} / \mathrm{mL})$, or Angll $(300 \mathrm{nM})$ was added to the upper chamber and incubated for another $24 \mathrm{~h}$. Thereafter, the cells were stained with a Diff Quik staining kit (Kobe, Japan), and those on the lower surface of the filter were photographed and counted under a fluorescence microscope. All treatments were performed in duplicate wells. The sequences of siHSPA7 are as follows: sense: UGAGC GUGACAGCCACUGACAGGAGCUU; antisense: GCUCCUGUCAGUGGCUGUC ACGC UCAUU.

\section{Enzyme-linked immunosorbent assay (ELISA)}

Cell culture supernatants were collected, and the amount of secreted IL-1 $\beta$ or IL-6 was quantified using ELISAs (R\&D Systems, Minneapolis, MN, USA) according to the manufacturer's protocol. Ninety-six-well plates were coated with $1 \mathrm{mg} /$ well capture antibody. The coated plates were washed twice with phosphate-buffered saline containing $0.05 \%$ Tween-20. Following exposure to the medium, the assay plates were exposed sequentially to each of the biotin-conjugated secondary antibodies. The plates were read at an absorbance of $450 \mathrm{~nm}$. Target proteins were analyzed according to the manufacturer's specification. Appropriate specificity controls were included, and all samples were run in duplicate.

\section{Quantitative real-time PCR (RT-qPCR)}

RNA was extracted from cells using a Ribospin RNA Extraction Kit (GeneAll, Seoul, Korea). The integrity of the extracted RNA was analyzed with a NanoDrop and quantified using spectrophotometric absorbance at $260 \mathrm{~nm}$. Next, $1 \mu \mathrm{g}$ of RNA was used for CDNA synthesis using an iScript $^{\text {TM }}$ cDNA Synthesis kit (Bio-Rad, Hercules, CA, USA). RT-qPCR was performed with a SYBR Green dye system on a LightCycler 480 real-time PCR machine (Roche, Basel, Switzerland) using a standard protocol. LightCycler software was used to analyze gene expression based on cycle threshold values normalized to $\beta$-actin expression. $\beta$-actin was used, as it is a commonly used housekeeper for normalization in RT$\mathrm{qPCR}^{17}$. Amplified gene expression was assessed by melting curve analysis, and no reverse transcriptase or template controls were included. Analyses were performed in duplicates. RT-qPCR was used to verify IncRNAs detected by RNA sequencing and SMC phenotype marker genes. PCR primers are as follows. F: CATCCAGGTGTATGAGGTTGAG. R: CACGCTCAGGATGCCATTA. The CNN1 and TAGLN genes encode calponin-1 and SM22a, respectively. These are markers of the contractile phenotype of SMCs. Inflammation is known to switch the vascular SMC phenotype from contractile to synthetic ${ }^{18}$.

\section{RNA-binding protein immunoprecipitation (RIP) assay}

The RIP assay was conducted using an EZ-Magna RIP kit (Merck Millipore, Burlington, MA, USA) according to the manufacturer's instructions. HASMCs were harvested and lysed in RIP lysis buffer. Cell extracts were then incubated with RIP buffer containing magnetic beads conjugated to anti-AGO2 antibody and IgG (Merck Millipore). Immunoprecipitated RNA was isolated, and GPCR analysis was performed to detect HSPA7 and miR-223. The $2^{-\triangle \Delta C t}$ method was used for quantification of RNAs, and $\mathrm{U} 6$ and $\beta$-actin were used as controls for miR-223 and HSPA7, respectively. 
1844

\section{Statistical analysis}

All data are presented as the mean \pm standard error of the mean. Analysis of variance, followed by Tukey's test, was used to compare values of continuous variables between groups with post hoc analysis. Differences were considered statistically significant when the $p$ value was $<0.05$. We used the software package Prism 5.0 for all data analyses (GraphPad Software, Inc., San Diego, CA, USA).

\section{RESULTS \\ Identification of IncRNAs associated with human atherosclerosis}

RNA sequencing was performed to identify IncRNAs associated with atherosclerotic plaques. A total of 380 RNAs were found to be differentially expressed between plaques and controls (Fig. 1a). Hierarchical clustering and multidimensional scaling were conducted based on fragments per kilobase of transcripts per million mapped reads values (fold change $>2 ; p<0.05$ ), and the gene expression pattern in plaques was distinct from that of the controls. Multidimensional scaling visualized differences in gene expression between the two groups (Fig. 1b). Figure 1c presents high-ranking IncRNAs with up- or downregulated expression in plaques. qPCR to confirm the differential expression of IncRNAs showed four with significantly up- or downregulated expression. Among them, two IncRNAs with upregulated expression, HSPA7 and LOC102724297, and one with downregulated expression, LINC00982, were selected for the validation experiment (Fig. 1d). When HASMCs were incubated with LPS, oxLDL, or Angll, only HSPA7 showed upregulated expression, whereas the expression of the other two IncRNAs was not substantially changed (Fig. 1e).

\section{Knockdown of HSPA7 attenuates migration and inflammatory changes in HASMCs}

To examine the effect of HSPA7 knockdown on HASMC migration, we transfected the cells with siHSPA7 or control siRNA for $24 \mathrm{~h}$ and plated them in the upper Transwell chamber with or without LPS, oxLDL, or Angll. After another $24 \mathrm{~h}$, analysis of the cells in the lower chamber revealed that migration of HASMCs promoted by oxLDL was significantly inhibited after siHSPA7 treatment (Fig. 2a, b). After transfection with siHSPA7 or control siRNA, HASMCs were treated with or without oxLDL for $24 \mathrm{~h}$. ELISAs and qPCR showed that the oxLDL-promoted secretion and expression of IL-1 $\beta$ and IL6 were suppressed by siHSPA7. The effect on IL- 6 was not notable (Fig. 2c). Immunofluorescence staining showed that the expression of markers of the contractile SMC phenotype, SM22a, and calponin1, was decreased upon oxLDL treatment, and this change was reversed by siHSPA7. The expression of CD68, a marker of macrophage-like cells, was upregulated upon oxLDL treatment, whereas this change was partly inhibited by siHSPA7 (Fig. 2d). The effects of HSPA7 on phenotype markers, particularly CNN1 and $C D 68$, were validated using qPCR (Fig. 2e).

\section{HSPA7 promotes inflammatory changes in HASMCs by sponging miR-223}

miRcode (http://www.mircode.org/) was used to search for candidate miRNAs interacting with HSPA7, and miR-223 had an optimal HSPA7 binding site. First, 17 miRNAs were shown to bind $H S P A 7$, and five miRNAs, miR-7/7ab, miR-96/507/1271, miR-182, miR-218/218a, and miR-223, that had higher conservation were selected. Among them, miR-182, miR-218/218a, and miR-223 were reported to affect IL-1 $\beta$ or IL-6 expression ${ }^{19-21}$. Finally, miR-223, which potentially affects the NLRP3 inflammasome ${ }^{21}$ and SMCs ${ }^{22}$ and has HSPA7 binding sites not overlapping with other miRNAs, was selected as a candidate miRNA. miRDB (http://mirdb.org/) revealed FOXO1 as a target of miR-223 (Fig. 3a). In addition, FOXO1 has been reported to regulate $\mathrm{Nf}-\mathrm{kb}^{23}$ and to influence smooth muscle cell proliferation ${ }^{24}$. Thus, we evaluated FOXO1 among targets of miR-223. HASMCs were transfected with a miR-223 inhibitor (Fig. 3b) and/or siHSPA7 or control siRNA and then treated with $50 \mu \mathrm{g} / \mathrm{mL}$ oxLDL for $24 \mathrm{~h}$. IL-1 $\beta$ and IL- 6 secretion increased upon miR-223 inhibitor treatment. The enhanced secretion of chemokines, particularly IL-6, was diminished with the addition of siHSPA7. These findings were verified using qPCR (Fig. 3c). After the same treatment of HASMCs, luciferase activity was measured after $48 \mathrm{~h}$. qPCR showed that upregulated expression of FOXO1, a transcriptional activator of NF-kB, by the miR-223 inhibitor was diminished after siHSPA7 treatment. Although luciferasereported NF-KB activity was elevated upon miR-223 inhibition, this parameter was attenuated upon HSPA7 knockdown (Fig. 3d). After the same treatment of THP-1 cell-derived macrophages, the secretion of IL-1 $\beta$ was increased with the miR-223 inhibitor, whereas it was decreased with siHSPA7. Although IL- 6 exhibited a similar tendency to IL-1 $\beta$, it was not significant. qPCR demonstrated up- and downregulation of the levels of IL1B, IL6, and FOXO1 according to each treatment (Fig. 3e). In immunofluorescence staining, SM22a and calponin 1 expression were not changed upon miR-223 inhibitor treatment, whereas the expression of these markers increased after the addition of SiHSPA7 (Fig. 3f). These findings were verified using qPCR, particularly that of TAGLN (Fig. 3g).

\section{HSPA7 targets miR-223 in an AGO2-dependent manner}

To evaluate whether HSPA7 is associated with miRNA ribonucleoprotein complexes (miRNPs), we conducted an RIP assay in HASMCs using an AGO antibody. AGO2 expression was not different in the cells irrespective of the presence of oxLDL. RIP assays showed that HSPA7 and miR-223 were enriched in the AGO2-containing miRNPs compared to the lgG immunoprecipitates. Furthermore, HSPA7 binding to $A G O 2$ was enhanced in the presence of oxLDL. To clarify the AGO2-dependent interaction between HSPA7 and miR-223, we transfected HASMCs with or without miR-223 inhibitor and then treated them with oxLDL. In a RIP assay, HSPA7 expression was significantly lower in the AGO2 immunoprecipitates in the cells treated with the miR-223 inhibitor (Fig. 3h).

\section{DISCUSSION}

Major findings of the current study include the following: (1) the expression of HSPA7, a IncRNA, was differentially upregulated in human atherosclerotic plaques and induced by oxLDL; (2) HSPA7 knockdown inhibited the migration of HASMCs and secretion and expression of inflammatory mediators, whereas it recovered the expression of contractile markers of the cells reduced upon oxLDL treatment. (3) Although miR-223 inhibition promoted the activity or secretion of inflammatory proteins in HASMCs, HSPA7 knockdown diminished these effects. (4) The impact of HSPA7 on miR223 was mediated in an AGO2-dependent manner. Notably, this study identified HSPA7 in human atheroma and elucidated its function, although previous studies have not revealed the expression or function of HSPA7 in vascular tissue. Our study not only reported the association of this IncRNA with atherosclerosis but also elucidated its biological effects on target vascular cells and the molecular mechanism of action. Furthermore, although the relationship between miR-223 and inflammation and vascular disease has been suggested, the current study first showed that miR-223 sponging is involved in the HSPA7 pathway.

Several heat-inducible $H S P 70$ genes have been reported in humans, and $H S P A 7$, also called $H S P 70 B$, is one of them. Similar to HSPA6, this gene is located on chromosome 1. Diverse expression levels of other HSPA genes have been shown in variable tissues, including blood, testis, and nerves ${ }^{25}$. Although HSPA7OB mRNA is expressed in response to heat shock, this gene was found to encode no functional protein a decade $\mathrm{ago}^{26}$. Studies on this gene have been extremely limited since ${ }^{27}$. Here, for the first time, we identified the differential expression of HSPA7 in human atheroma and validated its effect on VSMCs.

In our study, HSPA7 affected VSMC status by sponging miR-223. miR-223 was reported to suppress the NLRP3 inflammasome in 


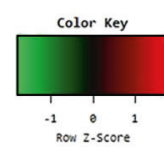

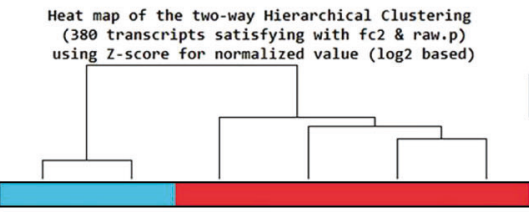

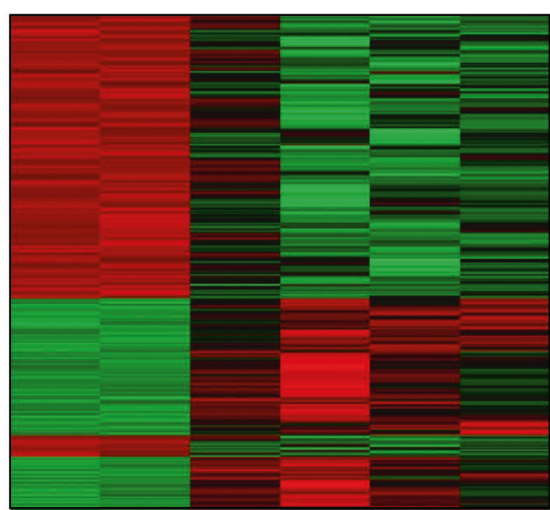

b
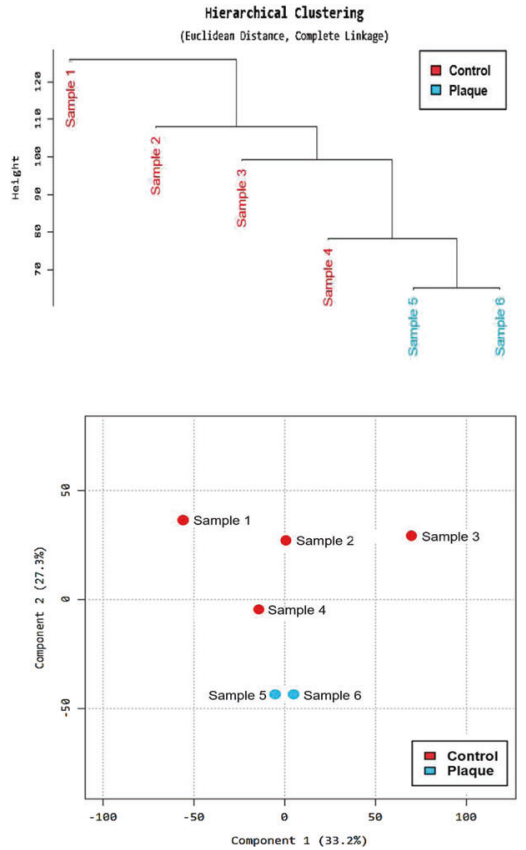

C

\begin{tabular}{|c|l|c|c|c|c|c|c|}
\hline \multirow{4}{*}{$\begin{array}{c}\text { UncRNA } \\
\text { accession }\end{array}$} & $\begin{array}{c}\text { Accession } \\
\text { number }\end{array}$ & $\begin{array}{c}\text { Chromo- } \\
\text { some }\end{array}$ & Start & Stop & $\begin{array}{c}\text { Fold } \\
\text { change }\end{array}$ & $p$ \\
\hline \multirow{4}{*}{\begin{tabular}{l} 
USulated \\
\cline { 2 - 8 }
\end{tabular}} & HSPA7 & NR_024151 & 1 & 161606291 & 161608217 & 6.13 & $4.50 \mathrm{E}-02$ \\
\cline { 2 - 9 } & LIPROBP & NR_033390 & 19 & 35904401 & 35908309 & 2.85 & $2.70 \mathrm{E}-02$ \\
\cline { 2 - 9 } & LOC102724297 & NR_073179 & 19 & 42397116 & 42409530 & 2.79 & $1.50 \mathrm{E}-03$ \\
\cline { 2 - 9 } & LAIR1 & NR_125406 & 3 & 46364660 & 46407059 & 2.18 & $4.64 \mathrm{E}-03$ \\
\cline { 2 - 9 } & SLC7A7 & NR_040448 & 14 & 22773222 & 22819811 & 2.04 & $3.91 \mathrm{E}-04$ \\
\hline \multirow{4}{*}{$\begin{array}{c}\text { Down- } \\
\text { regulated }\end{array}$} & NAV2-AS6 & NR_015384 & 11 & 19710934 & 19714672 & 2.86 & $1.00 \mathrm{E}-03$ \\
\cline { 2 - 9 } & LINC00312 & NR_024065 & 3 & 8571782 & 8574668 & 2.40 & $1.44 \mathrm{E}-02$ \\
\cline { 2 - 9 } & LINC00982 & NR_015440 & 1 & 3059617 & 3067725 & 2.37 & $1.00 \mathrm{E}-03$ \\
\cline { 2 - 9 } & MIR4697HG & NR_024344 & 11 & 133896435 & 133901740 & 2.07 & $1.88 \mathrm{E}-03$ \\
\hline
\end{tabular}

d
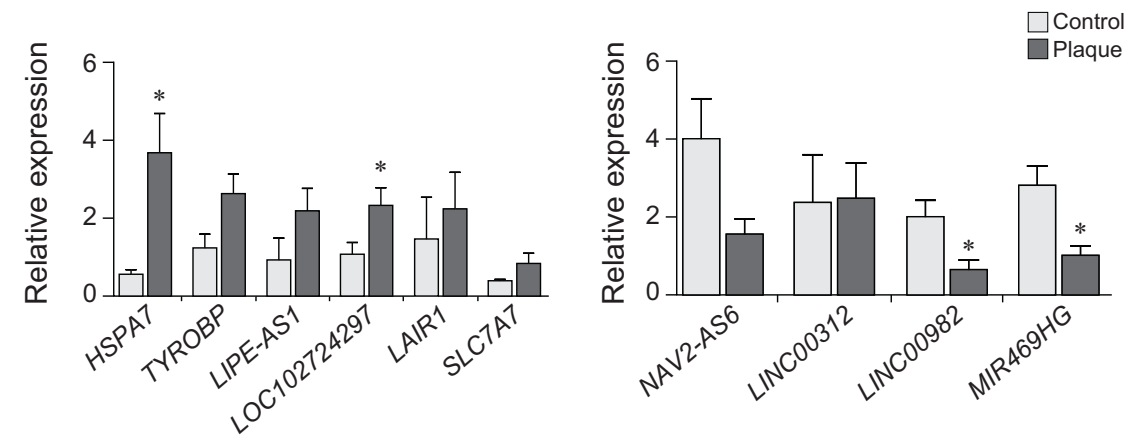

e
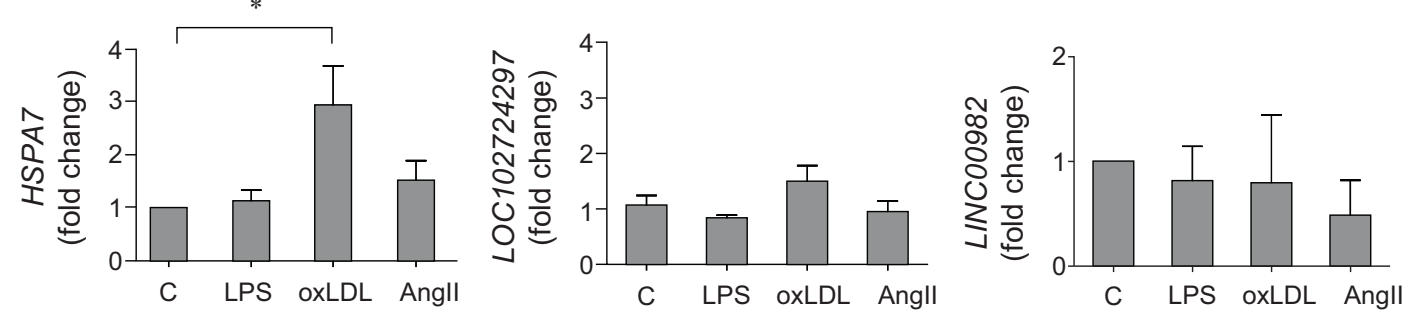

Fig. 1 HSPA7 expression is upregulated in human atherosclerotic plaques and induced by atherogenic stimuli. a Heatmap of genes differentially expressed in human atherosclerotic plaques compared to control tissues. $\mathbf{b}$ Hierarchical clustering and multidimensional scaling of data from plaques and controls. $\mathbf{c}$ List of high-ranking IncRNAs with up- or downregulated expression in plaques. $\mathbf{d}$ qPCR was performed to confirm IncRNAs with up- or downregulated expression using plaque and control tissues and four IncRNAs with significant differences. e Among the three top-ranked genes, only HSPA7 showed a significant elevation upon proatherogenic stimuli, particularly oxLDL. Experiments were conducted in technical duplicates, and data are presented based on three independent replicates. ${ }^{*}: p<0.05$. $\mathrm{C}$ control; oxLDL oxidized low-density lipoprotein. 
a

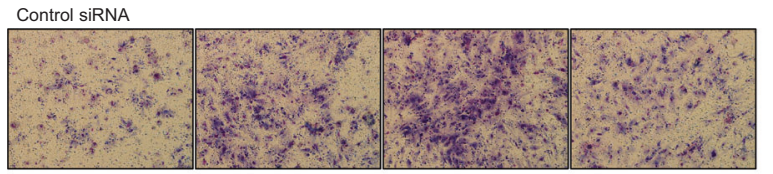

SIHSPA7

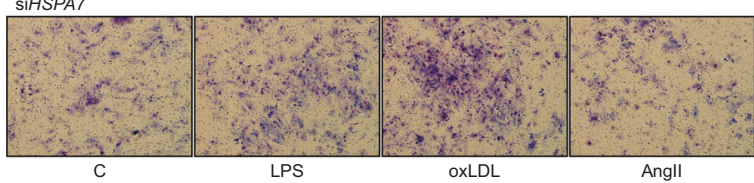

C

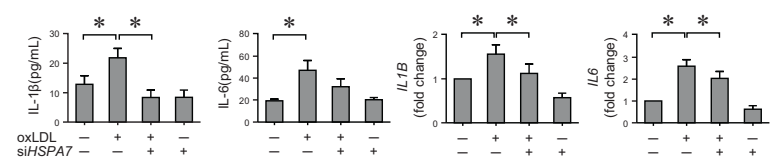

d
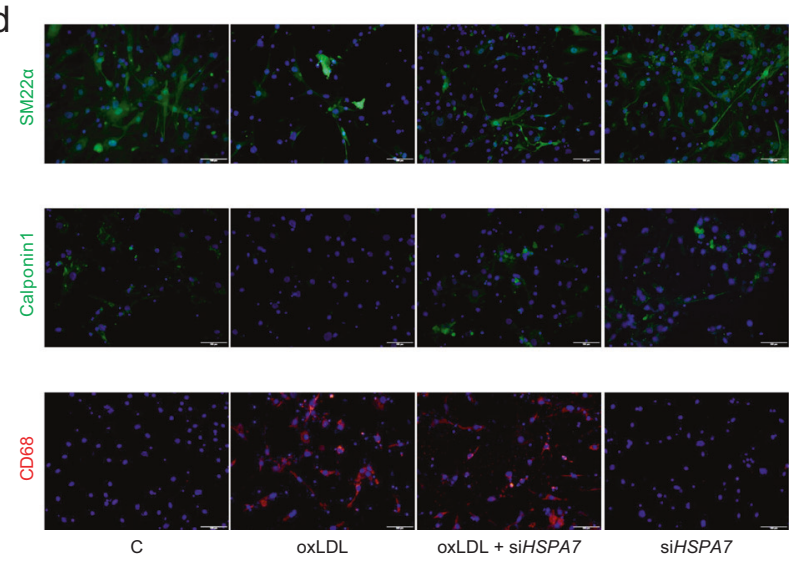

b

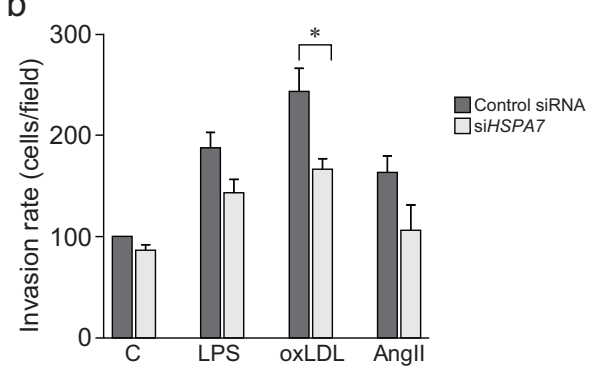

e
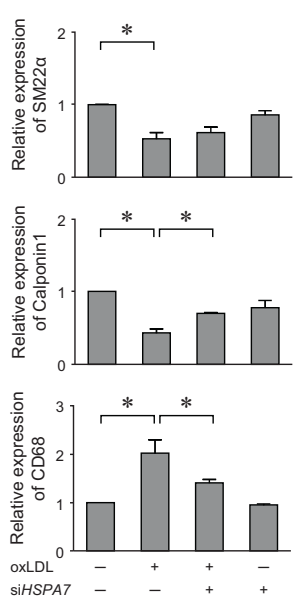
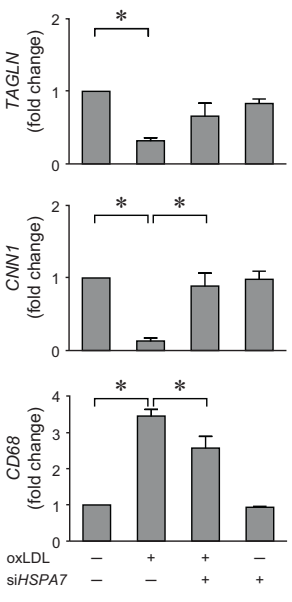

Fig. 2 Knockdown of HSPA7 attenuates migration and inflammatory changes in HASMCs. a, b Transwell assay for cell migration. HASMCs were transfected with siHSPA7 or scrambled siRNA for $24 \mathrm{~h}$ and plated in the upper chamber with or without LPS, oxLDL, or Angll. The cells in the lower chamber were stained and counted. Knockdown of HSPA7 significantly inhibited the oxLDL-induced migration of HASMCs. c ELISA and QPCR analyses showed that secretion and expression of IL-1 $\beta$ and IL- 6 promoted upon oxLDL treatment were suppressed by siHSPA7. $\mathbf{d}$, e Immunofluorescence staining revealed that SM22 $\alpha$ and calponin 1 were reduced upon oxLDL treatment, and this change was reversed by siHSPA7. The expression of CD68 upregulated by oxLDL was partly inhibited by siHSPA7. The effect of HSPA7 on phenotype markers, particularly CNN1 and CD68, was validated using qPCR. Data are from at least three independent experiments. ${ }^{*}: p<0.05$. C control; LPS lipopolysaccharide; oxLDL oxidized low-density lipoprotein; Angll angiotensin II; HASMC human aortic smooth muscle cell; ELISA enzymelinked immunosorbent assay; qPCR quantitative real-time polymerase chain reaction.

myeloid cells, and miR-223 dysregulation contributes to the pathogenesis of inflammatory diseases ${ }^{28}$. In addition, when miR223 expression is upregulated in endothelial cells, inflammatory pathways are inhibited ${ }^{21}$. Furthermore, miR-223 knockdown in mice increased atherosclerotic lesions, which indicates the protective role of miR-223 in vascular disease ${ }^{22}$. A recent study conducted in patients with Kawasaki disease showed that miR-223 increases VSMC differentiation and that miR-223 deficiency causes VSMC dedifferentiation ${ }^{29}$. These findings also support that changes in miR-223 expression may be involved in the development of atherosclerotic vascular disease. Because we demonstrated that miR-223 was a target of HSPA7 in atherosclerotic lesions, miR-223 inhibition by HSPA7 might contribute to the progression of atherosclerosis. Moreover, our findings on miR-223 and VSMCs suggested that miR-223 might be an appropriate target for cardiovascular prevention or a candidate disease marker. Most importantly, our study identified that HSPA7 is a regulator of miR-223 in the atherogenic vascular environment. Although regulation of FOXO1 by miR-223 that is affected by HSPA7 was shown in our data, the relationship between miR-223 and FOXO1 was previously reported. In more than one cancer cell line, overexpression of miR-223 downregulated FOXO1 expression and suppressed tumor cell proliferation ${ }^{30}$.
Here, we additionally showed the molecular mechanism of how HSPA7 exerts its effects. However, to date, detailed action mechanisms have been elucidated for just a few IncRNAs in VSMCs ${ }^{4,5}$. Lnc-Ang362 is regulated when VSMCs stimulated with angiotensin II act as hosts for the transcript of miR-221 and miR222 , and it is also involved in VSMC proliferation ${ }^{31}$. LncRNA-p21 reportedly inhibits VSMC proliferation and induces apoptosis, while its effect is associated with transcriptional activation of $\mathrm{p} 53^{9}$. SMILR (smooth muscle-induced IncRNA enhances replication) also regulates VSMC proliferation with the acceleration of the cell cycle by interacting with CENPF (centromere protein F) mRNA $^{32}$. In contrast, it is only incompletely understood how other IncRNAs affect VSMCs, such as ANRIL, SENCR, HAS-AS1, and H19,5.

In previous studies, IncRNAs involved in atherosclerosis have been discovered in mouse cells, as in the case of Mexis ${ }^{33}$. In addition, they were identified via genetic analysis even though the studies were performed in humans, similar to the case of $A N R L^{34}$. Otherwise, these IncRNAs were found in nonplaque human cells, as in the case of SENCR ${ }^{35}$. Studies identifying IncRNAs in human atherosclerotic plaque have been extremely rare, except for one study by Hung et al. that found PELATON using human carotid plaques ${ }^{36}$. The strength of our study is that we discovered HSPA7 in human plaques and elucidated the relevant biological function. 
a

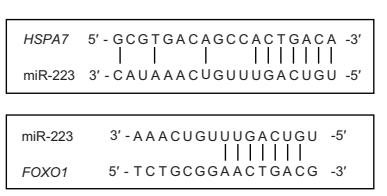

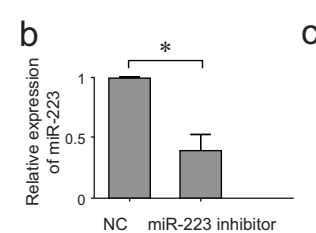

C
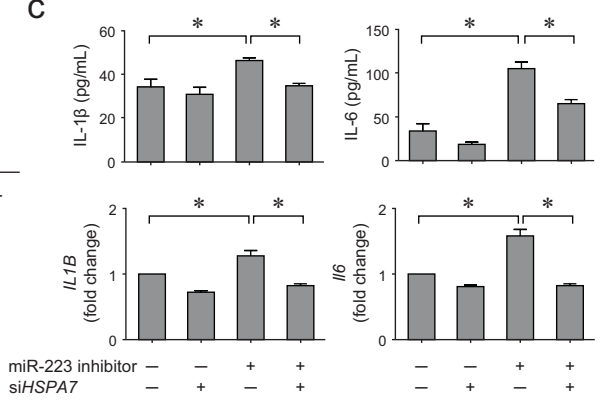

a

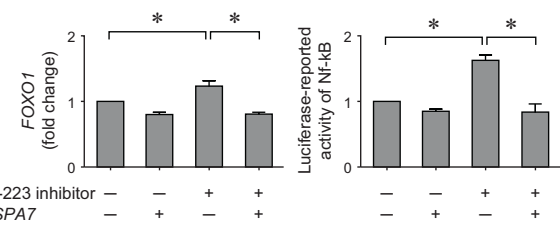

e
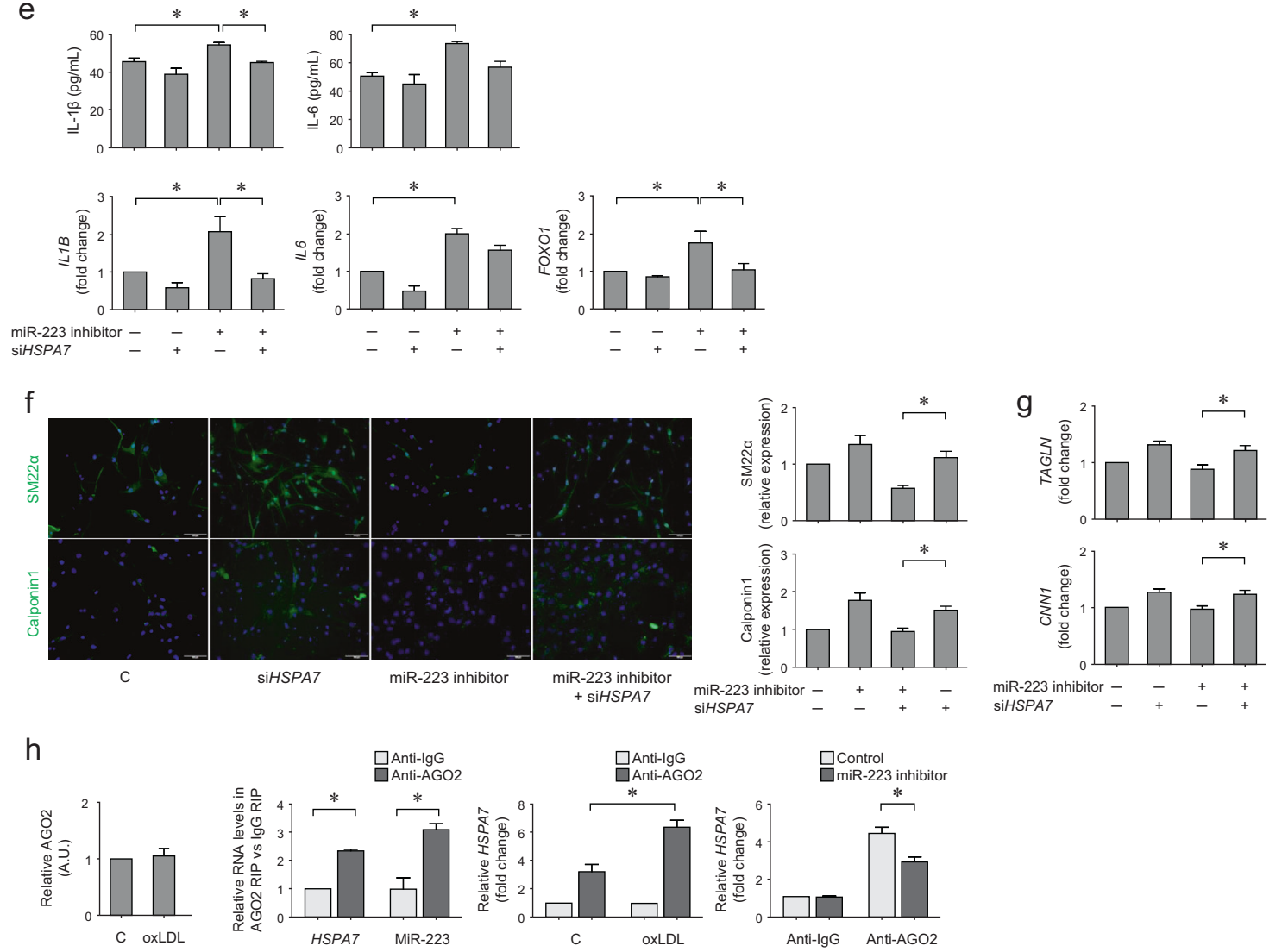

Fig. 3 HSPA7 promotes inflammatory changes in HASMCs by sponging miR-223. a Bioinformatics analysis using miRcode predicted that HSPA7 contains a potential miR-223 binding site. miRDB revealed that FOXO1 was a target of miR-223. b After transfection of HASMCs with miR-223, qPCR showed significantly downregulated miR-223 expression. c HASMCs were transfected with a miR-223 inhibitor and/or siHSPA7 or control siRNA and then treated with oxLDL. The secretion of IL-1 $\beta$ and IL- 6 was increased upon miR-223 inhibitor treatment, whereas it was diminished after siHSPA7 treatment, particularly for IL-6. These findings were verified using qPCR. d After the same treatment of HASMCs, qPCR showed that upregulated expression of FOXO1 induced by the miR-223 inhibitor was diminished after siHSPA7 treatment. Although luciferase-reported NF-KB activity was elevated upon miR-223 inhibition, this parameter was attenuated by SiHSPA7. e After the same treatment of THP- 1 cell-derived macrophages, the secretion of IL-1 $\beta$ and IL- 6 was measured and showed a similar tendency to that in HASMCs. qPCR demonstrated up- and downregulated expression of the corresponding genes and FOXO1.f Immunofluorescence staining showed that the expression of markers of the contractile SMC phenotype did not change upon miR-223 inhibitor treatment; however, they increased after siHSPA7 treatment. $\mathbf{g}$ These findings were verified using qPCR for TAGLN and CNN1. h miR-223 is a target of HSPA7 in an AGO2-dependent manner. AGO2 expression was not different in HASMCs regardless of the presence of oxLDL. RIP assays showed that HSPA7 and miR-223 were enriched in AGO2-containing miRNPs compared to IgG immunoprecipitates. HSPA7 had increased binding to AGO2 in the presence of oxLDL. HASMCs were transfected with or without miR-223 inhibitor and then treated with oxLDL. HAPA7 expression was shown to be significantly lower in AGO2 immunoprecipitates in the cells treated with the miR-223 inhibitor. Data are from at least three independent experiments. $*: p<0.05$. HASMC human aortic smooth muscle cell; oxLDL oxidized low-density lipoprotein; C control; AGO argonaute-2; RIP RNA immunoprecipitation; miRNP miRNA ribonucleoprotein.

Our study has potential limitations. We validated that HSPA7 promoted the inflammatory transition of VSMCs by sponging miR223. However, we cannot conclude that sponging miR-223 is the only mechanism of action for HSPA7, and additional studies may help elucidate the mechanism further. LncRNAs are poorly conserved across species compared to microRNAs, and mice do not have an HSPA7 homolog. Therefore, it was difficult to use a mouse model for the additional verification of the effect of HSPA7. 
1848

However, as mentioned, its discovery in human plaque and simultaneous elucidation of its mechanism of action have made our results scientifically meaningful.

In conclusion, HSPA7, a IncRNA identified in human atherosclerotic plaques, plays a role in the inflammatory transition of VSMCs stimulated by oxLDL. This process is mediated by miR-223 sponging by HSPA7. Notably, our study newly identified a IncRNA of previously unknown function in human plaques and elucidated its molecular mechanism of action. The current study suggests that HSPA7 can be a potential therapeutic target for atherosclerotic vascular disease.

\section{DATA AVAILABILITY}

The data underlying this article are available on reasonable request to the corresponding author.

\section{REFERENCES}

1. Lloyd-Jones, D. M. et al. Parental cardiovascular disease as a risk factor for cardiovascular disease in middle-aged adults: a prospective study of parents and offspring. JAMA 291, 2204-2211 (2004).

2. McPherson, R. \& Tybjaerg-Hansen, A. Genetics of coronary artery disease. Circ. Res. 118, 564-578 (2016).

3. Congrains, A. et al. Genetic variants at the $9 \mathrm{p} 21$ locus contribute to atherosclerosis through modulation of ANRIL and CDKN2A/B. Atherosclerosis 220, 449-455 (2012).

4. Pierce, J. B. \& Feinberg, M. W. Long noncoding RNAs in atherosclerosis and vascular injury: pathobiology, biomarkers, and targets for therapy. Arterioscler. Thromb. Vasc. Biol. 40, 2002-2017 (2020).

5. Fasolo, F., Di Gregoli, K., Maegdefessel, L. \& Johnson, J. L. Non-coding RNAs in cardiovascular cell biology and atherosclerosis. Cardiovasc. Res. 115, 1732-1756 (2019).

6. Song, J. \& Kim, Y. K. Discovery and functional prediction of long non-coding RNAs common to ischemic stroke and myocardial infarction. J. Lipid Atheroscler. 9, 449-459 (2020).

7. Enchill, Z., Lantz, C. \& Thorp, E. B. Select macrophage noncoding RNAs of interest in cardiovascular disease. J. Lipid Atheroscler. 9, 153-161 (2020).

8. Lv, J. et al. Long noncoding RNA H19-derived miR-675 aggravates restenosis by targeting PTEN. Biochem. Biophys. Res. Commun. 497, 1154-1161 (2018).

9. Wu, G. et al. LincRNA-p21 regulates neointima formation, vascular smooth muscle cell proliferation, apoptosis, and atherosclerosis by enhancing p53 activity. Circulation 130, 1452-1465 (2014).

10. Zhang, Z., Salisbury, D. \& Sallam, T. Long noncoding RNAs in atherosclerosis: JACC review topic of the week. J. Am. Coll. Cardiol. 72, 2380-2390 (2018).

11. Ono, K. et al. Functional non-coding RNAs in vascular diseases. FEBS J. https://doi. org/10.1111/febs.15678 (2020).

12. Lu, Y., Thavarajah, T., Gu, W., Cai, J. \& Xu, Q. Impact of miRNA in atherosclerosis. Arterioscler. Thromb. Vasc. Biol. 38, e159-e170 (2018).

13. Virmani, R., Kolodgie, F. D., Burke, A. P., Farb, A. \& Schwartz, S. M. Lessons from sudden coronary death: a comprehensive morphological classification scheme for atherosclerotic lesions. Arterioscler. Thromb. Vasc. Biol. 20, 1262-1275 (2000).

14. Liu, J. et al. Jointly defining cell types from multiple single-cell datasets using LIGER. Nat. Protoc. 15, 3632-3662 (2020).

15. Kim, G. S. et al. Impact of angiotensin II receptor blockers on clinical outcomes after percutaneous coronary intervention in patients with acute myocardial infarction based on data from the Korean National Health Insurance database (2005-2014). Korean Circ. J. 50, 984-994 (2020).

16. Jeong, S. J. et al. Deficiency of peroxiredoxin 2 exacerbates angiotensin II-induced abdominal aortic aneurysm. Exp. Mol. Med. 52, 1587-1601 (2020).

17. Kim, S. W. et al. Oleuropein prevents the progression of steatohepatitis to hepatic fibrosis induced by a high-fat diet in mice. Exp. Mol. Med. 46, e92 (2014).

18. Gomez, D. et al. Smooth muscle cell phenotypic switching in atherosclerosis. Cardiovasc. Res. 95, 156-164 (2012).

19. Liang, Q. et al. miR-182-5p attenuates high-fat-diet-induced nonalcoholic steatohepatitis in mice. Ann. Hepatol. 18, 116-125 (2019).

20. Li, F. et al. miR-218 impedes II-6-induced prostate cancer cells proliferation and invasion via suppression of LGR4 expression. Oncol. Rep. 35, 2859-2865 (2016).

21. Shi, X. et al. Paeonol inhibits NLRP3 mediated inflammation in rat endothelial cells by elevating hyperlipidemic rats plasma exosomal miRNA-223. Eur. J. Pharmacol. 885, 173473 (2020).

22. Shan, Z. et al. An endocrine genetic signal between blood cells and vascular smooth muscle cells: role of microRNA-223 in smooth muscle function and atherogenesis. J. Am. Col. Cardiol. 65, 2526-2537 (2015).

23. Lu, J. et al. The role of the FOXO1/ $\beta_{2}-A R / p-N F-K B$ p65 pathway in the development of endometrial stromal cells in pregnant mice under restraint stress. Int. J. Mol. Sci. 22, 1478 (2021).
24. Dutzmann, J. et al. BET bromodomain-containing epigenetic reader proteins regulate vascular smooth muscle cell proliferation and neointima formation. Cardiovasc. Res. 117, 850-862 (2021)

25. Daugaard, M., Rohde, M. \& Jaattela, M. The heat shock protein 70 family: highly homologous proteins with overlapping and distinct functions. FEBS Lett. 581, 3702-3710 (2007).

26. Parsian, A. J. et al. The human Hsp70B gene at the HSPA7 locus of chromosome 1 is transcribed but non-functional. Biochim. Biophys. Acta 1494, 201-205 (2000).

27. Brocchieri, L., de Macario, E. C. \& Macario, A. J. hsp70 genes in the human genome: Conservation and differentiation patterns predict a wide array of overlapping and specialized functions. BMC Evol. Biol. 8, 19 (2008).

28. Bauernfeind, F. et al. NLRP3 inflammasome activity is negatively controlled by miR-223. J. Immunol. 189, 4175-4181 (2012).

29. Zhang, Y. et al. Reduced platelet miR-223 induction in Kawasaki disease leads to severe coronary artery pathology through a miR-223/PDGFR $\beta$ vascular smooth muscle cell axis. Circ. Res. 127, 855-873 (2020).

30. $\mathrm{Wu}, \mathrm{L}$. et al. MicroRNA-223 regulates FOXO1 expression and cell proliferation. FEBS Lett. 586, 1038-1043 (2012).

31. Leung, A. et al. Novel long noncoding RNAs are regulated by angiotensin II in vascular smooth muscle cells. Circ. Res. 113, 266-278 (2013).

32. Mahmoud, A. D. et al. The human-specific and smooth muscle cell-enriched IncRNA SMILR promotes proliferation by regulating mitotic CENPF mRNA and drives cell-cycle progression which can be targeted to limit vascular remodeling. Circ. Res. 125, 535-551 (2019).

33. Sallam, T. et al. Transcriptional regulation of macrophage cholesterol efflux and atherogenesis by a long noncoding RNA. Nat. Med. 24, 304-312 (2018).

34. Kathiresan, S. et al. Genome-wide association of early-onset myocardial infarction with single nucleotide polymorphisms and copy number variants. Nat. Genet. 41, 334-341 (2009).

35. Bell, R. D. et al. Identification and initial functional characterization of a human vascular cell-enriched long noncoding RNA. Arterioscler. Thromb. Vasc. Biol. 34, 1249-1259 (2014).

36. Hung, J. et al. Novel plaque enriched long noncoding RNA in atherosclerotic macrophage regulation (PELATON). Arterioscler. Thromb. Vasc. Biol. 40, 697-713 (2020).

\section{ACKNOWLEDGEMENTS}

We are grateful to Jeong-Ho Kim, MD, PhD, for his help in the preparation of oxLDL and Medical Illustration \& Design, part of the Medical Research Support Service of Yonsei University College of Medicine, for all artistic support related to this work.

\section{AUTHOR CONTRIBUTIONS}

S.J.A. performed experiment, analyzed, and interpreted data, and wrote manuscript. H.B. and J.K.C. analyzed the data and revised manuscript. C.J.L., J.O., S.P., and S.M.K. interpreted the data and revised manuscript. S.H.L. conceptualized the study, acquired fund, administrated and supervised project, and wrote manuscript.

\section{FUNDING}

This work was supported by National Research Foundation of Korea grant funded by the Korean government (2019R1F1A1057952) and a faculty research grant of Yonsei University College of Medicine (6-2018-0037). The funder had no role in the design and conduct of the study; collection, management, analysis, and interpretation of the data; preparation, review, or approval of the manuscript; and decision to submit the manuscript for publication.

\section{COMPETING INTERESTS}

The authors declare no competing interests.

\section{ADDITIONAL INFORMATION}

Correspondence and requests for materials should be addressed to Sang-Hak Lee.

Reprints and permission information is available at http://www.nature.com/ reprints

Publisher's note Springer Nature remains neutral with regard to jurisdictional claims in published maps and institutional affiliations. 
Open Access This article is licensed under a Creative Commons Ac) Attribution 4.0 International License, which permits use, sharing,
adaptation, distribution and reproduction in any medium or format, as long as you give appropriate credit to the original author(s) and the source, provide a link to the Creative Commons license, and indicate if changes were made. The images or other third party material in this article are included in the article's Creative Commons license, unless indicated otherwise in a credit line to the material. If material is not included in the article's Creative Commons license and your intended use is not permitted by statutory regulation or exceeds the permitted use, you will need to obtain permission directly from the copyright holder. To view a copy of this license, visit http://creativecommons. org/licenses/by/4.0/.

(c) The Author(s) 2021 